\title{
Automated Analysis and Quantification of Human Mobility using a Depth Sensor
}

\author{
Daniel Leightley*, Member, IEEE, Moi Hoon Yap, Member, IEEE, and Jamie S.McPhee
}

\begin{abstract}
Analysis and quantification of human motion to support clinicians in the decision-making process is the desired outcome for many clinical-based approaches. However, generating statistical models that are free from human interpretation and yet representative is a difficult task. In this work, we propose a framework that automatically recognises and evaluates human mobility impairments using the Microsoft Kinect One depth sensor. The framework is composed of two parts. Firstly, it recognises motions, such as sit-to-stand or walking 4 metre, using abstract feature representation techniques and machine learning. Secondly, evaluation of the motion sequence in the temporal domain, by comparing the test participant with a statistical mobility model, generated from tracking movements of healthy people. To complement the framework, we propose an automatic method to enable a fairer, unbiased approach to label motion capture data. Finally, we demonstrate the ability of the framework to recognise and provide clinically relevant feedback to highlight mobility concerns, hence providing a route towards stratified rehabilitation pathways and clinician led interventions.
\end{abstract}

\section{INTRODUCTION}

$\mathbf{T}$ HERE is a clear advantage to developing automated systems to detect human motion for applications associated with healthcare [1]. The general population is living longer, therefore factor in providing health and social care services is to quantify and continuously assess people's health. While many people remain healthy, active and engaged into later life, studies have indicated that a minority suffer from frailty and musculo-skeletal mobility disorders [2]. Frailty is not a single disease; but a combination of the natural ageing processes, during which neuromuscular systems decline, and the accumulation of medical conditions, which leaves a patient vulnerable to illness or trips and falls [3]. Frailty is an indicator of general health and well-being, and is usually assessed by asking the person to perform several standardised tests (e.g. walk back and forth, sit-to-stand) during which a clinician observes the activity for stability, duration, coordination and posture control.

Although direct clinical assessment is vital, there is a need to construct more efficient clinical approaches to address limitations in assessment [3]-[5]. First, clinician-led assessments are dependent upon the skills, experiences and opinion of

D. Leightley was with the School of Computing, Mathematics and Digital Technology, Manchester Metropolitan University. He is currently at Centre for Military Health Research, King's College London, e-mail: dleightley@ieee.org.

M. H. Yap is with the School of Computing, Mathematics and Digital Technology, Manchester Metropolitan University.

J. S. McPhee is from the School of Healthcare Science, Manchester Metropoli$\tan$ University.

Manuscript received XX X, X; revised X X, XX. the clinician, so are not always objective. Second, clinical assessments are open to subjective bias and variance between assessments as well as interpretation of the results. Third, the entire process can be time consuming considering the patients' need to attend the appointment to undertake the assessment and the need for clinics to arrange appointments and oversee the assessments. Fourth, people with mobility impairments increase their risk of further trauma by having to attend specialist clinics, so it would be preferable to undertake the assessment at home. Fifth, patients may exhibit different behaviour as a result of the examination, which may alter the outcome and perceptions by the clinician.

Several studies [6], [7] indicate that reliable detection of a person with "poor mobility" can predict future declines in health, meaning that early identification could enable earlier remedial clinician-led interventions [2], [8]. Several attempts have been made to develop home-based monitoring systems for assessment and rehabilitation [7], [9]. While these systems have been validated and have the potential to support homebased monitoring of mobility, they fall short of assessing for mobility impairments. Further, these systems provide a single health indicator whereas an in-depth descriptive indicator could prove more useful to a clinician. In addition, existing systems have been evaluated using game-orientated datasets, and without clinical validation [5], [6], [10]. We utilise the newly released K3Da dataset [11], which contains clinically relevant motions to validate our proposed framework.

In this work, we propose a reliable and non-invasive framework to recognise, assess and quantify the mobility of participants. The system acquires motion capture (MoCap) from a single depth sensor, where the skeletal stream is decomposed into a set of novel joint-group features. Analysis techniques are employed to provide joint-group feedback highlighting the state of mobility, hence providing detailed insight for clinicians. To identify and label the MoCap data, we propose a method for automating the ground truth labelling of MoCap that is free from human bias or interpretation.

This paper is structured as follows: Section II presents and discusses current literature. Section III describes our proposed method to recognising, decomposing and analysing human motion. Section IV demonstrates the effectiveness of our method for classifying and analysing human motion. Finally, Section $\mathrm{V}$ provides a discussion, concludes our work and presents future avenues of research.

\section{RELATED WORK}

Several works have been proposed to measure and analyse human motion and stability. The most prevalent methodology 
suggests using one or a combination of intrusive sensors, such as body-based accelerometer or markers. Then, the clinical relevant indicators are extracted by analysing the patterns presented in time-series data. In recent years, the computer vision community has proposed an array of solutions to solve this challenge, but majority are not validated clinically. These works have predominantly focused on depth sensor technology, removing the need for intrusive sensors, which has been shown to be sufficiently accurate and responsive for tracking in both in-home and clinical settings [10], [12], [13].

A number of frameworks have been proposed to enable greater understanding of human motion. These frameworks follow a similar structure. They first seek to identify the motion, using recognition frameworks, and then undertake quantitative analysis [14], [15]. The Rehabilitation Gaming System [9], [16], [17] extracts the stability of a participant using a combination of gloves and markers on the hands. After the participant performs several standardised tests, the data is processed to calculate compliance. Dolatabadi et al. [18] proposed a home-based system for assessing changes in gait and balance. The authors utilise a Microsoft Kinect 360 (Kinct 360) for $x B o x$ sensor to observe gait recovery in a participant that had undergone surgery. They were able to track the gait, and temporal change over a number of weeks. Gonzalez et al. [19] proposed a solution for real-time balance estimation by deriving Centre-of-Mass (CoM) feature from the Kinect 360 and a Wii Balance Board. The authors unite the CoM and angular momentum to quantify the stability of participant. While this work presents a novel solution to providing balance and stability measurements, it has been tested only on two participants.

There are few approaches that seek to unite recognition and quantitative analysis of human motion in a hierarchical context. Cary et al. [20] proposed a system to unite the Kinect 360 and Artificial Neural Network (ANN) to aid in recognition of physiotherapy assessment sessions. The authors design a feature vector based on joint groups. The first group is composed of the torso joints; with the second group the remaining joints. The vector is computed by extracting the associated angles between joints. The work employs a multilevel ANN that decomposes each joint into a separate model. This allows the recognition of complex motion sequences and assesses their correctness in relation to a predefined model. Kargar et al. [21] utilised a depth sensor to automatically measure the physical mobility of participants. They analyse and classify human gait in relation to "Get-up-and-Go-Test". Two types of features are extracted from the MoCap data provided by the sensor. The first type of feature is related to the human gait (e.g. number of steps, duration of each step, and turning duration), whereas the second type describes the anatomical configuration. The authors state that using these features provided a descriptor for characterising physical mobility. To enable classification of the imbalance severity, the authors implement a Support Vector Machine (SVM).

While works exist to assess human balance and gait, the work is limited to extracting single-valued indicators which lack clinical validation. To the best of the authors' knowledge, this work is the first proposed method to detect, analyse and provide clinically relevant outcome measures using depth sensor technology. Our work relies on several key computer vision and pattern recognition techniques: stereo vision, pose estimation, feature representation, clustering and temporal segmentation.

\section{APPLICATION FRAMEWORK}

The framework is divided into three parts. First, joint-group features are generated from skeletal MoCap data. Second, recognition of human motion using a range of machine learning classifiers to provide the clinician with the motion being observed. Finally, a motion analysis approach to providing clinically relevant outcome measures alongside the motion being evaluated is proposed.

\section{A. Feature Encoding}

Identification and recognition of motions is not a trivial task. Wang et al. [6] used feature groups to detect human action with a Kinect 360, which yielded promising results. However, the same feature set would not provide the abstract level of detail required for suitable clinical outcome measures. This, in part is due to the way in which motion performance between humans differs slightly, making a single top-level outcome generalised in nature [13], [22]. Derived features have been shown to be more useful than raw MoCap data [23]-[25]. This lead to the work of Du et al. [26], using a Hierarchical Recurrent Neural Network for human action recognition, at the core was the concept of dividing the skeleton into joint groups, based on anatomical significance to the motion sequence. A joint level group of features is encoded, representative of multiple motion types and shows promise in encoding subtle variations. As in [26], we employ the same joint group decomposition. A summary of the feature groups and encoding methodology is presented in Table I, with a visual illustration presented in Figure 1.

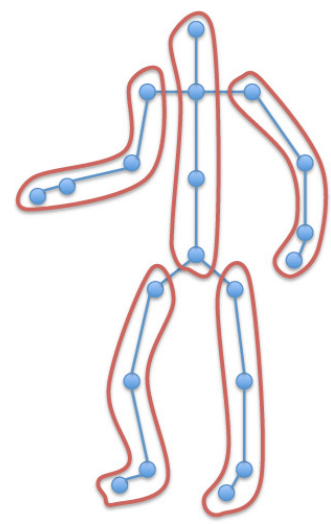

Fig. 1. An illustration of a MoCap skeleton divided into five joint groups as proposed in [26]. Each joint group represents a key motion area which is capable of representing all types of human motion.

There are multiple measurements which are capable of being extracted from a skeletal stream of MoCap [21], [25], [27]. The difficulty is to select the most appropriate features capable of describing the motion and subtle variations. Alongside these 
TABLE I

SUMMARY OF JOINT DECOMPOSITION AND DERIVED FEATURES FOR EACH GROUP AND THE CORRESPONDING DIMENSIONALITY OF THE FINAL FEATURE VECTOR. *Where $I$ is the number of features

\begin{tabular}{|c|c|c|c|}
\hline Joint Group & Features & Length & Notation* \\
\hline $\begin{array}{l}\text { Left Arm (LeftShoulder, LeftElbow, } \\
\text { LeftWrist, LeftHand) }\end{array}$ & $\begin{array}{l}\text { Left arm Euler Angle (between left shoulder and left wrist), } \\
\text { Euclidean distance between the left shoulder and left hand, } x \\
\text { and } y \text { axis vectors. }\end{array}$ & 12 & $F_{\text {LeftArm }}=\{1 \ldots, I\}$ \\
\hline $\begin{array}{l}\text { Left Leg } \\
\text { LeftAnkle, LeftFoot) }\end{array}$ & $\begin{array}{l}\text { Left leg Euler Angle (between left hip and left ankle), } \\
\text { Euclidean distance between the left hip and left foot, } x \text { and } \\
y \text { axis vectors. }\end{array}$ & 12 & $F_{\text {LeftLeg }}=\{1 \ldots, I\}$ \\
\hline $\begin{array}{lr}\text { Right Arm } & \text { (RightShoulder, } \\
\text { RightElbow, } & \text { RightWrist, } \\
\text { RightHand) } & \end{array}$ & $\begin{array}{l}\text { Right arm Euler Angle (between right shoulder and right } \\
\text { wrist), Euclidean distance between the right shoulder and } \\
\text { right hand, } x \text { and } y \text { axis vectors. }\end{array}$ & 12 & $\begin{array}{l}F_{\text {Right Arm }} \\
\{1 \ldots, I\}\end{array}$ \\
\hline $\begin{array}{l}\text { Right Leg (RightHip, RightKnee, } \\
\text { RightAnkle, RightFoot) }\end{array}$ & $\begin{array}{l}\text { Right leg Euler Angle (between right hip and right ankle), } \\
\text { Euclidean distance between the right hip and right foot, } x \\
\text { and } y \text { axis vectors. }\end{array}$ & 12 & $F_{\text {RightLeg }}=\{1 \ldots, I\}$ \\
\hline $\begin{array}{l}\text { Torso (SpineBase, SpineMid, Neck, } \\
\text { Head, SpineShoulder) }\end{array}$ & $\begin{array}{l}\text { Torso Euler Angle (between the spin base and neck) relative } \\
\text { to the body, Euclidean distance between the spine base and } \\
\text { head, Body lean angle (relative to the floor with torso as } \\
\text { a reference), Centre-of-Mass (between left shoulder, right } \\
\text { shoulder, spine mid), } x \text { and } y \text { axis vectors. }\end{array}$ & 15 & $F_{\text {Torso }}=\{1 \ldots, I\}$ \\
\hline
\end{tabular}

derived features, raw MoCap data itself is also utilised. The $x$ and $y$ coordinates are extracted for each joint to describe the posture change with respect to the axis [23].

Euler Angle: Any rigid body can be described as an angle around three orthogonal coordinates in fixed space. However, computing Euler Angles from marker-less MoCap is difficult. Joint angles are extracted using the approach by Lewandowski et al. [28]. Frames are normalised, and then three angles defining the reference joint (see Table I) are computed and represented by a three unit angle $\left(\mathbb{R}^{3}\right)$.

Euclidean Distance: An important characteristic of human motion is the way in which the participant transitions over time in relation to a fixed point. For example, with the Torso Group, the Euclidean distance is computed between the base of the spine and head. While this value will remain constant for motions such as walking, when the participant performs a bend, or sit-to-stand the distance between the two joints differs. The change in distance is computed as:

$$
\text { distance }=\sqrt{\left(x_{1}-x_{2}\right)^{2}+\left(y_{1}-y_{2}\right)^{2}+\left(z_{1}-z_{2}\right)^{2}}
$$

where $x_{1}, y_{1}, z_{1}$ and $x_{2}, y_{2}, z_{2}$ are the reference joints.

Body Lean Angle: It is possible to represent a rotation group (SO $(3))$, which is a rotation in Euclidean space as a set of two vectors (unit vector $\hat{\mathbf{e}}$ indicating the direction of lean, $\theta$ angle representing the magnitude of rotation about the axis). The Body Lean Angle represents the body in relation to the ground plane, see Figure 2. The angle is computed by the flexion of the spine in relation to ground floor plane, defined as the centre of the feet. The lean angle between the spine and the floor is defined as:

$$
\hat{\mathbf{e}}, \theta=\arccos \left(\frac{S \cdot Q}{\|S\| Q \|}\right)^{t}
$$

where $S$ is the spine vector $(x, y, z)$ and $Q$ is the floor vector - the centre of the feet $(x, y, z)$.

Centre-of-Mass: The CoM [19], [29] is extracted from MoCap data to describe the directional movement. Figure 3 demonstrates a 2D visual example for the CoM for two motion

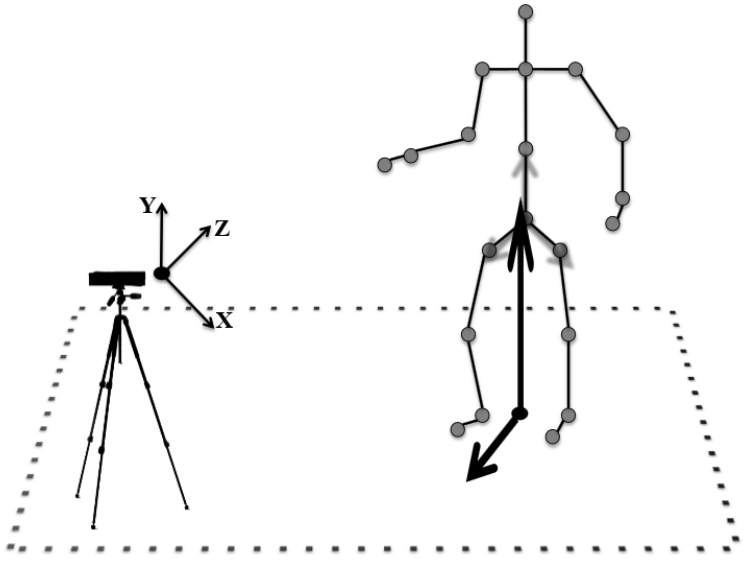

Fig. 2. Visual representation of the Body Lean Angle in relation to the Microsoft Kinect sensor. The angle is computed by the intersection between the ground plane and spine.

sequences. Vertical directional movements, such as Chair Rise, are identified by the Kinect One due to its ability to track millimetre postural changes [30]. In order to evaluate and measure stability, it is necessary to measure the movement of the body's CoM. Let com be the CoM at time $t$ of pose $p$ computed from three joints (hip left, hip right, spine) is given as:

$$
\begin{aligned}
& \bar{x}=\frac{\sum_{i=1}^{3} p_{t, x_{i}}^{\prime}}{i} \\
& \bar{y}=\frac{\sum_{i=1}^{3} p_{t, y_{i}}^{\prime}}{i} \\
& \bar{z}=\frac{\sum_{i=1}^{3} p_{t, z_{i}}^{\prime}}{i} \\
& \operatorname{com}=[\bar{x}, \bar{y}, \bar{z}]^{t}
\end{aligned}
$$

where $\bar{x}, \bar{y}, \bar{z}$ is the mean, $i$ is the joint index of frame $t$ and com is the concatenation of the mean values. 

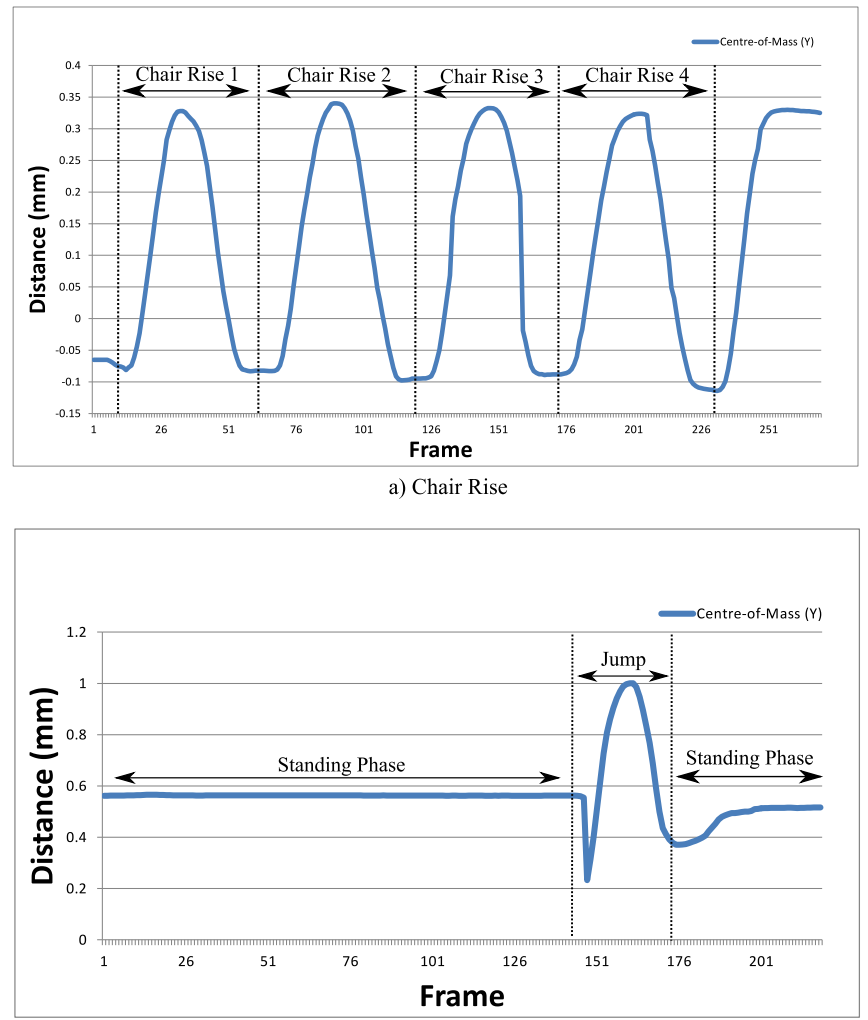

b) Maximum Jump

Fig. 3. Visual 2D representation of the CoM for two motion types. a) CoM ( $y$ axis) for Chair Rise. b) CoM ( $y$ axis) for Maximum Jump.

\section{B. Recognition: Motion Identification}

The framework for recognising trials (otherwise referred to as motions) is shown in Figure 4. Recognition enables the clinician to be aware of the motion performed by the participant. The framework is divided into two aspects. First, offline training of multiple machine learning classifiers based on an exemplar-based pose selection. Second, online detection and identification of motions in real-time.

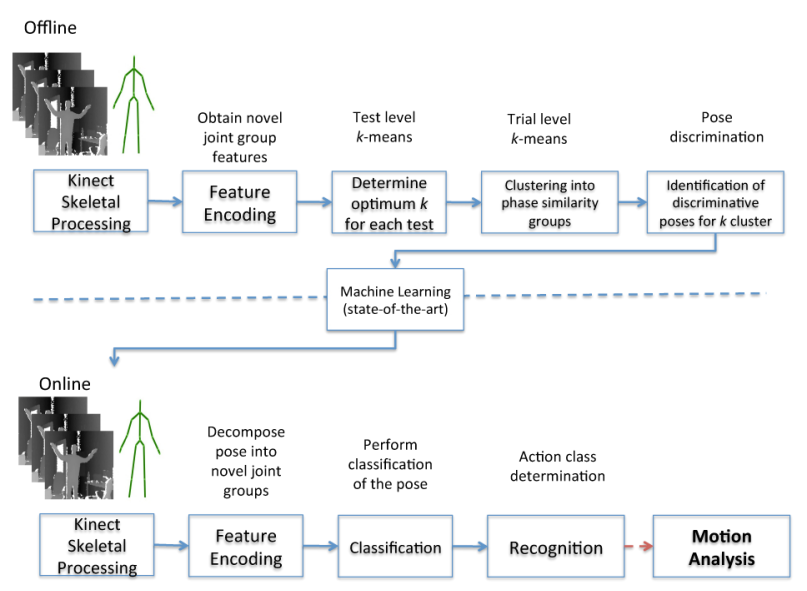

Fig. 4. Recognition Overview: Top row illustrates the training process. Bottom row illustrates the online recognition process.

1) Feature Reduction and Selection: For generalisation and consistency, $\mathcal{P}$ represents all skeletal poses, ordered in a time-sequential manner. Each feature encodes a motion characteristic, such as gait, or motion performance. To train any machine learning classifier, a unified training sample needs to be formed, given as:

$$
\begin{array}{r}
\hat{F}=\left\{F_{\text {LeftArm }}, F_{\text {LeftLeg }}, F_{\text {RightArm }},\right. \\
\left.F_{\text {RightLeg }}, F_{\text {Torso }}\right\} \in \mathbb{R}^{63}
\end{array}
$$

where $\hat{F}$ is a combined vector consisting of the features derived for each joint group.

The objective is to identify and extract only those features that contain enough descriptive information to describe the motion. A two tier clustering process is employed (Algorithm 1 illustrates the pseudo code). Top-level process combines the features for each trial into a single matrix. An automated clustering approach is then employed to identify the optimum number of clusters. $k$-means has been selected over other approaches as it is computationally faster when dealing with a large number of observations. Further, it is suitable for grouping and segmenting human motion compared hierarchical clustering methods. Then, sub-level clustering is undertaken on each feature group with the derived optimum clusters. This results in a feature set $\hat{F}$, is represented by $k$ clusters. The next stage is to identify and extract key features. The clustering process results in a dynamic number of $k$ clusters of any length. A "key" cluster is identified if it contains more than $N / K$, the average size of the clusters. For each key cluster, features are retained using an equivalence function. 


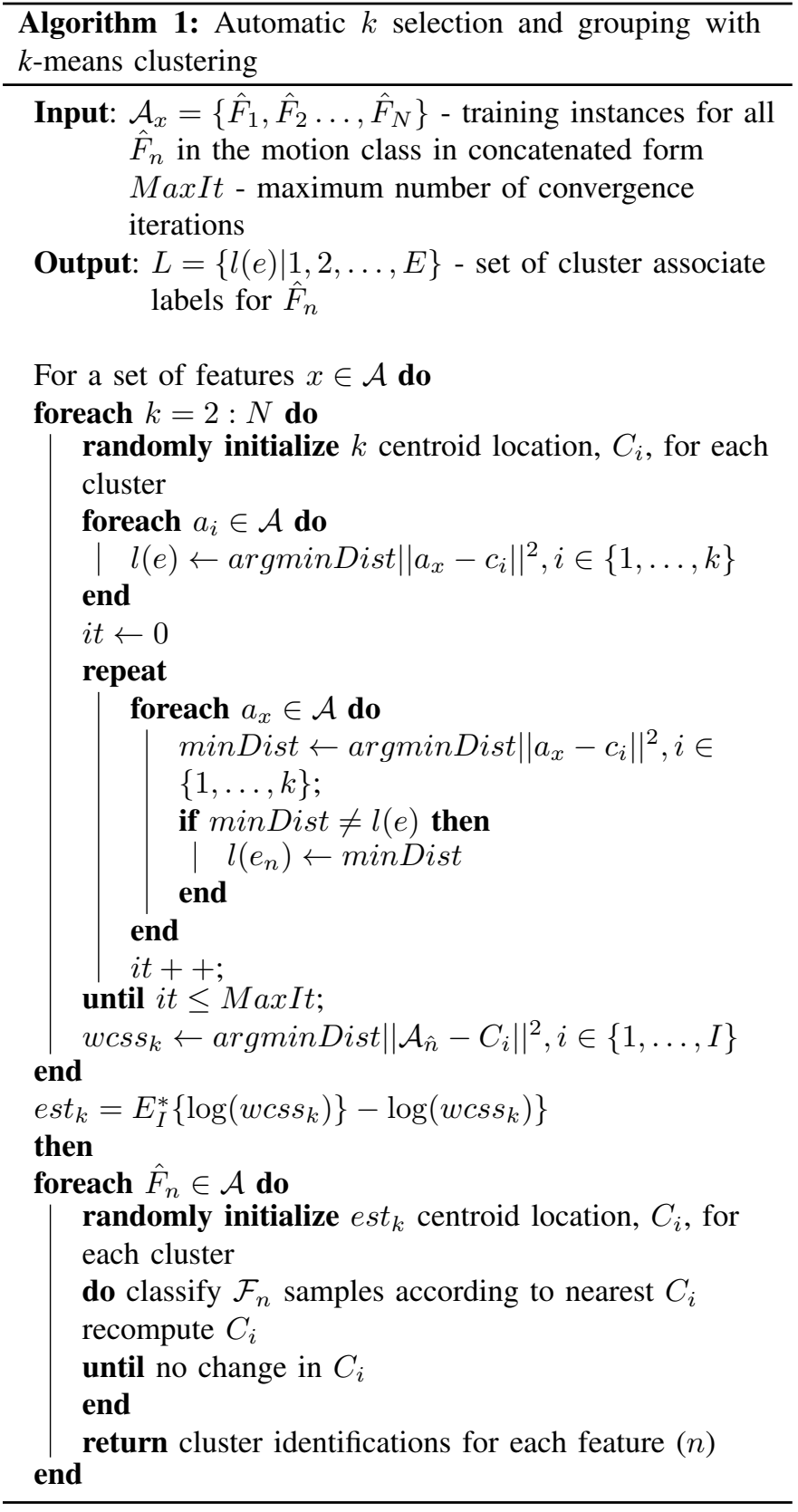

The similarity between two features, $a$ and $b$ from $\hat{F}$ is computed as:

$$
\operatorname{Similarity}\left(\hat{F}_{a}, \hat{F}_{b}\right)=\min \left\|a_{i}-b_{j}\right\|^{2}
$$

A Self-Similarity Matrix $S$ for a key cluster $K C$ from $\hat{F}$ can be computed using Eq. 5 and defined as:

$$
S:=\left(s_{i, j}\right)_{N_{z} x N_{z}}=\left\{\operatorname{Similarity}\left(\hat{F}_{i}, \hat{F}_{j}\right)\right\}_{N_{z} x N_{z}} \in K C
$$

where $S$ is the computed Similarity-Matrix with a dimensionality of $N_{z} \times N_{z}$ for cluster $K C$. The median element of the Similarity-Matrix $S_{\text {median }}$ is selected, and a cost function is defined to identify features that are within a threshold, denoted as hold are retained. This is computed as:

$$
D\left(S_{\text {median }}, S_{i}\right)=\text { hold } \Varangle \sum_{i=1}^{I}\left\|S_{\text {median }}-S_{i}\right\|^{2}
$$

where $i \in I=\{1,2, \ldots, I\}$ is the number of poses for each key cluster and $D$ are the features which fall within the threshold hold. Hence, only those that are shown to be informative are retained. This provides a reduced feature vector.

2) Recognition: For recognition, several machine learning classifiers are evaluated (Table II). To train a model, each action class is represented by a set of key clusters with finetuning and parameter selection undertaken to improve the model stability. To classify, the skeletal stream is encoded in real-time using the features summarised in Table I, each pose is provided to the model for assignment of a class predictor.

\section{Motion Analysis}

The framework for analysing human mobility is presented in Figure 5. The framework is split into two parts. First, MoCap is assigned a ground truth marker to identify if it contains "good" or "poor" mobility, then multiple SVMs are trained to detect mobility change. Second, identification and analysis of participants mobility is undertaken to provide clinically relevant outcome measures. In the recognition stage, the joint groups are merged into a single feature vector, whereas for motion analysis each joint group is trained as a separate model.

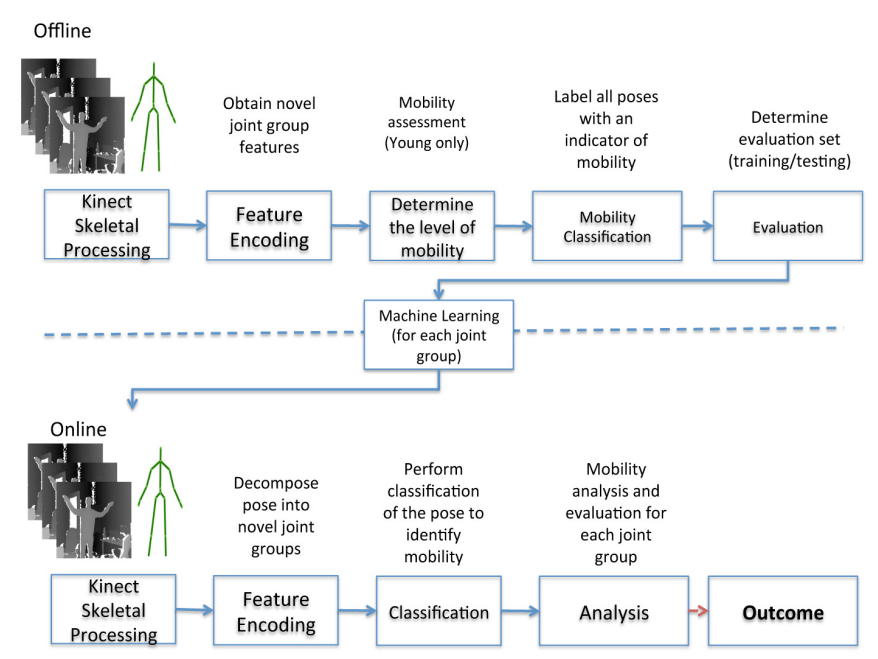

Fig. 5. Motion Analysis Overview: Top row illustrates the process undertaken to label, group and train a set of SVM models. Bottom row illustrates the analysis approach utilised to provide clinically relevant outcome measures.

1) Labelling and Computation of Human Mobility: For a typical recognition task, prior knowledge of the class label is required. This is usually straightforward to determine, for example a person walking or jumping can easily be defined with a single label [1]. However, the task becomes very difficult to identify and label in the context of different styles of the same motion. For example, attempting to group different types of gait manually can result in subjective grouping and 
TABLE II

LIST OF MACHINE LEARNING CLASSIFIERS UTILISED, INCLUDING FINE-TUNING/PARAMETER SELECTION METHODOLOGY.

\begin{tabular}{|l|l|l|}
\hline Machine Learning & $\begin{array}{l}\text { Parameters } \\
\text { Required }\end{array}$ & Parameters Selection Methodology \\
\hline Support Vector Machines (SVM) [31] & $C$ and $\gamma$ & Parameter selection was undertaken using cross-validation method. \\
\hline Random Forests (RF) [32] & $n_{\text {tree }}$ and $m_{t r y}$ & $\begin{array}{l}n_{\text {tree }} \text { selection is undertaken using cross-validation method iterating } \\
\text { from } n_{\text {tree }}=100 \text { to } n_{\text {tree }}=5,00 \text {. and } m_{\text {try }} \text { set at a default of 3. }\end{array}$ \\
\hline Artificial Neural Networks (ANN) [32] & $\begin{array}{l}n_{\text {layer }}, \text { neurons, } \\
\text { rate }\end{array}$ & $\begin{array}{l}\text { Parameter selection was undertaken using stratified } k \text {-fold cross vali- } \\
\text { dation with a validation set employed to present over-training. }\end{array}$ \\
\hline $\begin{array}{l}\text { Gaussian Restricted Boltzmann Machines (GRBM) } \\
\text { [33] }\end{array}$ & $h_{\text {variable }}$ & The number of hidden units, $h_{\text {variable } \text { was set at a default of 500. }}$ \\
\hline Adaptive Boosting (AB) [34] & $n_{\text {iterations }}$ & Parameter selection was undertaken using cross-validation. \\
\hline LPBoost [35] & $n_{\text {iterations }}$ & Parameter selection was undertaken using cross-validation. \\
\hline RUSBoost [36] & $n_{\text {iterations }}$ & Parameter selection was undertaken using cross-validation. \\
\hline Total Boost (TB) [37] & $n_{\text {iterations }}$ & Parameter selection was undertaken using cross-validation. \\
\hline Bagging [38] & $n_{\text {tree }}$ & Parameter selection was undertaken using cross-validation. \\
\hline
\end{tabular}

bias [18], [39]. There have been several approaches proposed to obtain and measure clinically supportive outcome(s), yet they have manually annotated motions with little clinically supportive reasoning for labelling [6], [13], [39]. However, there are methods for objectively identifying and grouping human motion in the clinical literature. Baumgartner et al. [40] introduced a normal distribution of motion values to derive the standard deviation (SD) of the mean to define groups of sarcopenia (loss of muscle mass with ageing). This has been used extensively within the medical community to identify different groups [3], [41], yet to the authors' knowledge it has not been utilised within the computer science community.

Our proposal extends [40] work to define "good" and "poor" mobility using a digitalised labelling framework, based on the data itself. This approach is free from human interpretation, bias or subjectiveness except for providing a threshold value. The labelling can be summarised as follows: Frames that contain a value greater than the $\pm 1.5 \mathrm{SD}$ of the mean are identified having "poor" mobility, whereas frames within \pm 1.5 SD of the mean are identified having "good" mobility. Each joint group is labelled individually. For each group, each individual frame is labelled as having "good" mobility or "poor" mobility. The labelling is summarised as follows:

1) Using young adults from the $K 3 \mathrm{Da}$ dataset, each motion class and joint group is combined into a single matrix. This results in five matrices representing each joint group.

2) The $\pm 1.5 \mathrm{SD}$ from the mean (computed as the average per feature row) is computed for each joint group.

3) Using the SD of the mean values computed at item 2, all trials including young and elderly from the K3Da dataset are labelled.

4) Frames that that are within the $\pm 1.5 \mathrm{SD}$ are labelled as having "good" mobility.

5) Frames that lie greater than $\pm 1.5 \mathrm{SD}$ are labelled as having "poor" mobility.

The mean $\pm 1.5 \mathrm{SD}$ threshold value is computed from the young only, to represent the general population. As this work seeks to identify mobility, using the elderly may create a bias within the model and increase the rate of false positives. Table III provides a summary for the number of frames labelled as having "good" or "poor" mobility for each participant group.
TABLE III

FRAME LABELS FOR "GOOD MOBILITY" AND "POOR MOBILITY" FOR EACH JOINT GROUP, REPRESENTED FOR EACH PARTICIPANT CATEGORY.

\begin{tabular}{|l|l|l|l|l|}
\hline Joint Group & Young & Old & \\
\hline & Good (\%) & Poor (\%) & Good (\%) & Poor (\%) \\
\hline Left Arm & $31,382(87)$ & $4,131(13)$ & $12,516(51)$ & $12,851(49)$ \\
\hline Right Arm & $32,145(84)$ & $5,146(16)$ & $13,728(54)$ & $11,639(46)$ \\
\hline Left Leg & $30,367(89)$ & $3,368(11)$ & $18,089(60)$ & $7,278(40)$ \\
\hline Right Leg & $31,344(87)$ & $4,169(13)$ & $19,725(72)$ & $5,642(28)$ \\
\hline Torso & $31,355(87)$ & $4,158(13)$ & $16,089(57)$ & $9,278(43)$ \\
\hline
\end{tabular}

The labelling of each frame provides information of the state of mobility at any period of time; A "mobility score" metric is derived to indicate the level of mobility the participant has compared to the statistical models derived earlier. The mobility score is an aggregate of the number of frames identified as having "good" mobility versus "poor" mobility for each joint group.

2) Analysing Mobility using Multiple SVMs: Over recent years, a number of classifiers have been proposed for the task of motion recognition, consistently SVM have yielded high accuracy results. They are computationally less expensive to train, and provide a low latency for recognition compared to others (e.g. Random Forest). The aim is to generate a detailed insight of a participant's mobility. To that end, a random sample of participants from the K3Da dataset are extracted, and each joint group is modelled using an SVM with 10-fold cross-validation, Figure 6 demonstrates the training and evaluation pipeline. While it is possible to train a single SVM, indeed [1], [42] obtained high accuracy results for the task of recognition. If we follow these type of approaches, subtle motion variations would be overshadowed resulting in over generalisation (over fitting) leading to inter-/intraclass confusion between "good" mobility and "poor" mobility. Training an individual SVM for each joint group models subtle changes in motion, providing a greater motion context. Furthermore, it permits the framework to identify specific joint groups that may be of concern.

To compute a clinical outcome measure, test data is decomposed into joint-group based features and provided to the corresponding SVM. Each SVM provides a feature-level classification for "good" mobility or "poor" mobility, detailing the state of mobility. Then, detailed analysis of the motion 


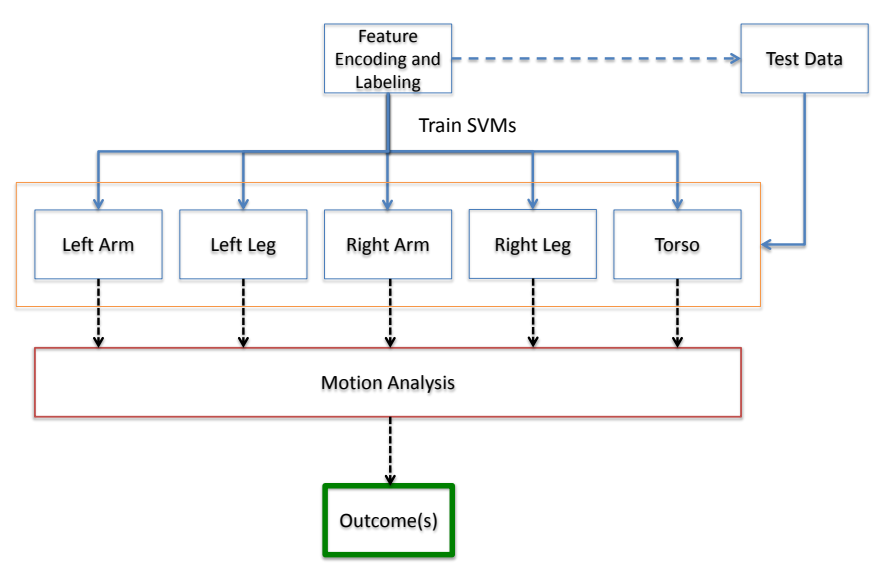

Fig. 6. Summary of the training and evaluation approach for analysing human mobility.

is undertaken on the complete labelled sequence to provide feedback on the participants mobility.

For practical utilisation, each joint group is assessed based on the number frames classified as having "good" mobility versus "poor" mobility. If any joint group has more than a predefined number of frames labelled as "poor" mobility, such as $30 \%$, an outcome is generated highlighting that further investigation is required.

\section{ExPERIMENTAL: Motion DeteCtion AND QUANTIFICATION}

The validation of the framework is undertaken in two parts. First, evaluate the ability of the framework to perform motion recognition. Second, evaluate the ability of the framework to detect mobility concerns and impairment. The evaluation is performed on "unseen" test participants, meaning that no test data has been included during the modelling phase. The K3Da Dataset [11], which contains 54 participants performing a wide range of clinically validated motions, captured using the Microsoft Kinect One sensor were used. We selected five trials scenarios from the dataset namely; balance (eyes open), chair rise, semi-tandem balance, tandem balance, walk (4 metre).

\section{A. Evaluation: Motion Detection}

We evaluated the performance of a range of machine learning classifiers using our joint groups. These classifiers, together with parameter selection methodology are presented in Table II. For each technique, a 10-fold cross-validation using the random 'leave-one-out' principle was used to compute recognition accuracy. Table IV illustrates the performance of each machine learning algorithm for motion recognition.

We were able to achieve acceptable recognition rates (Table IV), particularly when we consider the similarity in motions using our proposed joint groups and features. Random Forests produced the highest average recognition rate of $87.10 \%$, GRBM producing the lowest average result of $73.08 \%$. The recognition rates fluctuated due to the leave-one-out approach employed. Encouragingly, similar motions such as semitandem and tandem balance were identifiable, with little inter/intra-class variation.

Below average recognition rates were observed for several iterations, this, in part due to the formation of the training and testing sets or cross-validation and parameter selection may have struggled due to inter-class similarity. Being able to correctly identify a motion is important to ensure the correct model is applied for motion analysis and quantification. Another consideration is the time in which it takes to perform classification, an average recognition per frame was below $1 \mathrm{~ms}$, suggesting real-time recognition is viable. Leightley et al. [11] reported a peak accuracy of $85.53 \%$, with our proposals obtaining a marginally increased peak accuracy of $87.10 \%$.

\section{B. Experimental: Motion Analysis}

The ground truth labels derived in this work are used to evaluate the proposed framework for the task of detecting mobility. Figure 7 provides an overview for the success of the overall framework in identifying features of concern in relation to the ground truths.

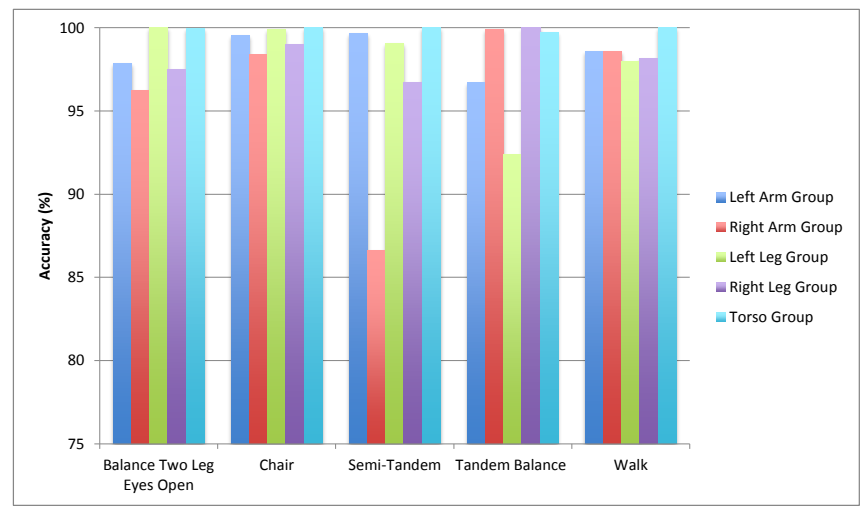

Fig. 7. Success of the framework when compared to ground truth labelling.

For evaluation, a random sample of participants were used for training and the remainder used for testing, using leaveone-out methodology. Across trials evaluated, a high truepositive rate is obtained (Figure 7). This indicates that the framework is capable of correctly identifying mobility concerns based on the ground truths.

We were able to detect a large number of participants who had been identified at labelling as possibly having a mobility concern. Overall, the confusion matrix for each joint group performed strongly, with a high rate of true positives, and a small rate of false positives with an overall sensitivity of 0.98 , specificity of 0.95 and Matthew Correlation Coefficient (MCC) score of 0.94 supporting this conclusion.

Balance - Two Legs (Eyes Open): Each participant stood with their feet as close together as possible side-by-side. They balanced with their eyes open and arms extended horizontally to be parallel with the floor. Each joint group could be correctly identified, with only Left Leg providing the lowest detection rate of $96.23 \%$. The framework detected large amounts of mobility concern, most notably in the Left Arm. 
TABLE IV

MACHINE LEARNING RECOGNITION RATE FOR EACH ITERATION AND CLASSIFIER.

\begin{tabular}{|c|c|c|c|c|c|c|c|c|c|}
\hline Iteration & SVM & RF & ANN & GRBM & AdaBoost & LPBoost & RUSBoost & Total Boost & Bagging \\
\hline 1 & $80.78 \%$ & $86.75 \%$ & $83.92 \%$ & $87.83 \%$ & $78.36 \%$ & $82.58 \%$ & $89.50 \%$ & $84.19 \%$ & $79.98 \%$ \\
\hline 2 & $79.94 \%$ & $82.85 \%$ & $78.38 \%$ & $81.01 \%$ & $86.22 \%$ & $88.12 \%$ & $85.15 \%$ & $87.92 \%$ & $82.47 \%$ \\
\hline 3 & $82.99 \%$ & $89.04 \%$ & $82.46 \%$ & $79.87 \%$ & $91.08 \%$ & $90.89 \%$ & $87.67 \%$ & $83.29 \%$ & $87.15 \%$ \\
\hline 4 & $76.59 \%$ & $93.48 \%$ & $80.92 \%$ & $82.10 \%$ & $92.13 \%$ & $84.76 \%$ & $82.73 \%$ & $84.77 \%$ & $89.86 \%$ \\
\hline 5 & $86.43 \%$ & $78.47 \%$ & $82.19 \%$ & $80.56 \%$ & $81.79 \%$ & $79.36 \%$ & $83.89 \%$ & $88.91 \%$ & $85.78 \%$ \\
\hline 6 & $76.49 \%$ & $83.67 \%$ & $70.71 \%$ & $82.37 \%$ & $79.37 \%$ & $83.85 \%$ & $83.74 \%$ & $86.07 \%$ & $84.37 \%$ \\
\hline 7 & $86.65 \%$ & $92.16 \%$ & $80.68 \%$ & $76.10 \%$ & $83.58 \%$ & $85.52 \%$ & $90.17 \%$ & $86.55 \%$ & $\mathbf{8 9 . 9 8 \%}$ \\
\hline 8 & $84.24 \%$ & $87.92 \%$ & $82.83 \%$ & $78.34 \%$ & $82.01 \%$ & $84.71 \%$ & $86.24 \%$ & $87.72 \%$ & $87.72 \%$ \\
\hline 9 & $81.05 \%$ & $90.11 \%$ & $74 \%$ & $56.28 \%$ & $74.18 \%$ & $86.62 \%$ & $85.71 \%$ & $88.23 \%$ & $88.47 \%$ \\
\hline 10 & $77.14 \%$ & $86.60 \%$ & $78.99 \%$ & $74.09 \%$ & $87.61 \%$ & $88.09 \%$ & $84.37 \%$ & $83.47 \%$ & $89.91 \%$ \\
\hline Average (SD) & $81.23 \%$ & $87.10 \%$ & $79.50 \%$ & $73.08 \%$ & $83.63 \%$ & $85.45 \%$ & $85.91 \%$ & $86.11 \%$ & $86.56 \%$ \\
\hline
\end{tabular}

TABLE V

OVERALL CONFUSION MATRIX FOR THE PERFORMANCE OF EACH JOINT GROUP IN IDENTIFYING MOBILITY CONCERNS. WHERE TRUE POSITIVE INDICATES THE PARTICIPANT HAS GOOD MOBILITY AND TRUE NEGATIVE INDICATES PARTICIPANTS WITH A MOBILITY CONCERN.

Left Arm - Predicted Outcome

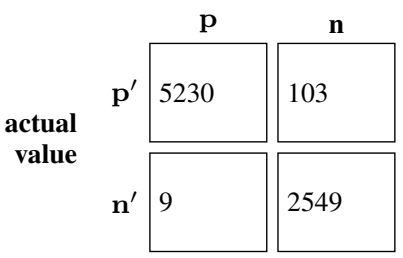

Right Arm - Predicted Outcome

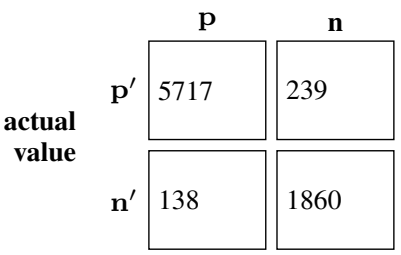

Left Leg - Predicted Outcome

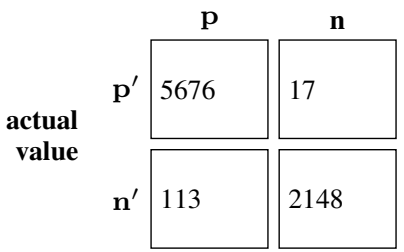

Right Leg - Predicted Outcome

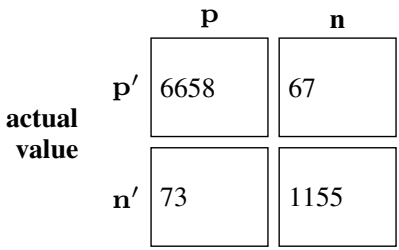

Torso - Predicted Outcome

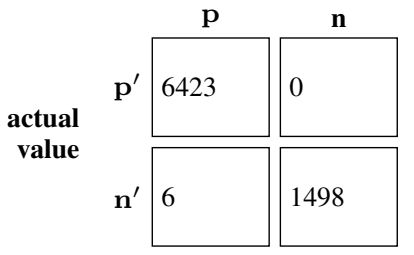

A sensitivity of 0.99 , a specificity of 0.96 and a MCC of 0.96 was obtained. Mobility was in line with expectations, with the framework performing reliably across all joint groups.

Chair Rise: Each participant started from a seated position. When promoted, they had to stand up so that the legs were fully extended, and then sit down again. This was repeated five times with the aim to complete five complete stand/seat cycles. The arms were held across the chest so that all of the power needed to stand and sit was produced by the legs muscles. Each joint group could be correctly identified, with only Left Leg providing the lowest detection rate of $98.38 \%$. A sensitivity of 0.99 , specificity of 0.96 and an MCC of 0.96 was obtained. Overall, mobility across the participant range was good, with only a minority of features falsely classified as having "concern" across the joint groups.
Semi-Tandem Balance: Each participant placed one foot behind the other so that the big toe of the back foot was touching the side of the heel of the front foot. Their arms were fully extended horizontally for a period of 10 seconds. Each joint group could be correctly identified, with only Right Arm providing the lowest detection rate of $86.60 \%$. A large number of features were identified as having a "concern" in Right Arm; this may be due to the incorrect recognition of features. This false classification is further observed with a relatively low sensitivity of 0.95 , a specificity of 0.99 and a MCC of 0.91 .

Tandem Balance: Each participant placed one foot directly behind the other so that the big toe of the back foot was touching the back heel of the front foot. The arms were fully extended horizontally for a period of 10 seconds. Each joint 
group could be as being correctly identified, with only Left Arm providing the lowest detection rate of $96.69 \%$. For Right $L e g$, all features were classified correctly. This may be in part due to most participants using their left leg for the balancing resulting in the sensor being obscured from the right leg; therefore the Right Joint Group may be hidden for duration of the motion. A high sensitivity of 0.99 was achieved, however low scores for specificity of 0.91 and MCC of 0.92 .

Walk (4 metres): Each participant started from a standing position and walked forwards in a straight line towards the sensor at their 'usual' walking speed. Each joint group could be correctly identified, with only Right Arm providing the lowest detection rate of $98.10 \%$. Across the joint groups, features were classified correctly, with only a few features being identified as a concern requiring further invention, this was further supported with a sensitivity of 0.99 . However, when considering specificity of 0.94 and a MCC of 0.95 , the framework does yield low results for this type of trial when compared to the others.

With the proposed framework it is possible to use the recognition of frames to identify if any intervention or rehabilitation is required. A threshold of $70 \%$ was selected through experimentation, if any joint group contained more than $70 \%$ of frames classified as "concern" it would be acknowledged as requiring investigation by a clinician. Of the participants used in these experiments, 16 were highlighted as having at least one joint group of concern. In a clinical context, these participants would be examined further by a clinician to determine why poor mobility was observed. In accuracy terms, this is a $94 \%$ success rate in detecting mobility concerns between participant groups based on ground truth labelling.

\section{Discussion AND CONCLUSION}

The release of the Kinect One has presented new capacities for innovation within the healthcare sector. The ability to deploy the sensor in a wide range of locations, as well as its low-cost are important highlights. Further, the Kinect One is capable of providing detailed measurements extracted from motion sequences and encoded features. Standard approaches, such as SPPB [43] provide a single score measurements with no contextual information whereas the Kinect One is able to provide finite kinematic information. The extraction of joint groups provides an abstract level of detail and insights into how the joint group is performing in relation to the motion as a whole, this leads to an improved insight for clinicians to make a recommendation.

In this work, we utilise the Kinect One for detecting mobility concerns to aid in stratified clinical intervention. The motions used for evaluation are commonplace and necessary parts of typical daily living, such as walking, sitting, standing and balancing. These same movements become problematic for persons with a mobility concerns of any age. Due to the large inter-individual variability in age and physical capabilities, the K3Da dataset is well suited for evaluating the proposed framework. However, it contain only a limited number of participants which makes general population modelling difficult. If the number of participants for modelling is increased, a more reliable and representative population model can be computed. Future work will explore this in more detail.

While analysis is a key theme of this work, it is important to consider detection of motions as they occur, this enables the correct outcome measure to be applied. We have compared several classifiers, presenting a detailed comparison of their ability to detect a range of subtly different motions. They have been able to detect subtle differences between similar motions, for example semi-tandem and tandem balance with the aid of our proposed feature set. This work has united recognition and motion analyse to provide a united decision making process.

We propose a framework which unites human motion recognition techniques with motion analysis. The framework has been shown to be reliable and accurate for evaluation of mobility. By utilising low-cost depth sensor technology the application framework is deployable in a large number of scenarios and environments, resulting in real world practical benefits. Future work will focus on clinical validation of the proposed framework with an increased population size using power analysis.

\section{REFERENCES}

[1] D. Leightley, J. Darby, B. Li, J. S. Mcphee, and M. H. Yap, "Human activity recognition for physical rehabilitation," in Systems, Man, and Cybernetics (SMC), 2013 IEEE International Conference on. IEEE, 2013, pp. 261-266.

[2] R. M. Collard, H. Boter, R. A. Schoevers, and R. C. Oude Voshaar, "Prevalence of frailty in community-dwelling older persons: a systematic review," Journal of the American Geriatrics Society, vol. 60, pp. 14871492, 2012.

[3] General Practitioners, "Fit for frailty: Consensus best practice guidance for the care of older people living with frailty in community and outpatient settings," British Geriatrics Society, Tech. Rep., 2014.

[4] M. Fuhrer, "Subjectifying quality of life as a medical rehabilitation outcome," Disability Rehabilitation, vol. 22, no. 11, pp. 481-489, 2000.

[5] P. Gregory, J. Alexander, and J. Satinksky, "Clinical telerehabilitation: Applications for physiatrists," American Academy of Physical Medicine and Rehabilitation, vol. 3, no. 7, pp. 647 -656, July 2011.

[6] R. Wang, G. Medioni, C. J. Winstein, and C. Blanco, "Home monitoring musculo-skeletal disorders with a single $3 \mathrm{~d}$ sensor," in Proceedings of the IEEE Conference on Computer Vision and Pattern Recognition Workshops, 2013, pp. 521-528.

[7] J. Bae and M. Tomizuka, "A tele-monitoring system for gait rehabilitation with an inertial measurement unit and shoe-type ground reaction force sensor," Mechatronics, 2013.

[8] L. P. Fried, C. M. Tangen, J. Walston, A. B. Newman, C. Hirsch, J. Gottdiener, T. Seeman, R. Tracy, W. J. Kop, G. Burke, and M. A. McBurnie, "Frailty in older adults: Evidence for a phenotype," Gerontology: Biological Sciences, vol. 56, no. 3, pp. 808-813, 2001.

[9] M. S. Cameirao, S. Bermudez, E. D. Oller, and P. F. Verschure, "The rehabilitation gaming system: a review," Studies in health technology and informatics, vol. 145, pp. 65- 83, 2009.

[10] D. Webster and O. Celik, "Experimental evaluation of microsoft kinect's accuracy and capture rate for stroke rehabilitation applications," in Haptics Symposium, Feb 2014, pp. 455-460.

[11] D. Leightley, M. H. Yap, Y. B. J. Coulson, and J. S. Mcphee, "Benchmarking human motion analysis using kinect one: an open source dataset," in IEEE Conference of Asia-Pacific Signal and Information Processing Association, 2015.

[12] R. Clark, Y. H. Pua, K. Fortin, C. Ritchie, K. Webster, L. Denehy, and A. Bryant, "Validity of the microsoft kinect for assessment of postural control," Gait and Posture, vol. 36, no. 3, pp. 372 - 377, 2012.

[13] B. Galnaa, G. Barrya, D. Jacksonb, D. Mhiripiria, P. Olivierb, and L. Rochestera, "Accuracy of the microsoft kinect sensor for measuring movement in people with parkinson's disease," Gait and Posture, vol. 39, no. 4, pp. 1062-1068, 2014.

[14] N. Vernadakis, V. Derri, E. Tsitskari, and P. Antoniou, "The effect of xbox kinect intervention on balance ability for previously injured young competitive male athletes: A preliminary study," Physical Therapy in Sport, vol. 15, no. 3, pp. 148-155, 2014. 
[15] S. Gauthier and A. Cretu, "Human movement quantification using kinect for in-home physical exercise monitoring," in IEEE Conference on Computational Intelligence and Virtual Environments for Measurement Systems and Applications, 2014.

[16] M. S. Cameirão, S. Bermúdez, E. D. Oller, and P. F. Verschure, "Neurorehabilitation using the virtual reality based rehabilitation gaming system: methodology, design, psychometrics, usability and validation," NeuroEngineering and Rehabilitation, vol. 7, no. 48, 2010.

[17] M. da Silva Cameirao, S. B. i Badia, E. Duarte, and P. F. Verschure, "Virtual reality based rehabilitation speeds up functional recovery of the upper extremities after stroke: A randomized controlled pilot study in the acute phase of stroke using the rehabilitation gaming system," Restor Neurol Neurosci, vol. 29, no. 5, pp. 287-298, Jan 2011

[18] E. Dolatabadi, B. Taati, G. S. Parra-Dominguez, and A. Mihailidis, "A markerless motion tracking approach to understand changes in gait and balance: A case study," in Rehabilitation Engineering and Assistive Technology Society of North America, 2013.

[19] A. Gonzalez, P. Fraisse, and M. Hayashibe, "A personalized balance measurement for home-based rehabilitation," in IEEE Conference on Neural Engineering, 2015, pp. 711-714.

[20] F. Cary, O. Postolache, and P. Silva Girao, "Kinect based system and artificial neural networks classifiers for physiotherapy assessment," in MeMeA, June 2014.

[21] A. Kargar, A. Mollahosseini, T. Struemph, W. Pace, R. Nielsen, and M. Mahoor, "Automatic measurement of physical mobility in get-upand-go test using kinect sensor," in IEEE Conference on Engineering in Medicine and Biology Society, 2014.

[22] V. Bloom, D. Makris, and V. Argyriou, "Clustered spatio-temporal manifolds for online recognition," in IEEE Conference on Pattern Recognition, August 2014, pp. 3963-3968.

[23] A. Sinha and K. Chakravarty, "Pose based person identification using kinect," in IEEE Conference on Systems, Man and Cybernetics, Oct 2013, pp. 497-503.

[24] E. Gianaria, N. Balossino, M. Grangetto, and M. Lucenteforte, "Gait characterization using dynamic skeleton acquisition," in International Workshop on Multimedia Signal Processing, Sept 2013, pp. 440-445.

[25] B. Dikovski, G. Madjarov, and D. Gjorgjevikj, "Evaluation of different feature sets for gait recognition using skeletal data from kinect," in $I n$ ternational Convention on Information and Communication Technology, Electronics and Microelectronics, 2014, pp. 1304-1308.

[26] Y. Du, W. Wang, and L. Wang, "Hierarchial recurrent neural network for skeleton based action recognition," in IEEE Conference on Computer Vision and Pattern Recognition, 2015, pp. 1110-1118.

[27] L. Zhang, J. C. Hsieh, T. T. Ting, Y. C. Huang, Y. C. Ho, and L. K. $\mathrm{Ku}$, "A kinect based golf swing score and grade system using gmm and svm," in CISP, 16-18 Oct 2012, pp. 711-715.

[28] M. Lewandowski, J. Martinez-Del-Rincon, D. Makris, and J. C. Nebel, "Temporal extension of laplacian eigenmaps for unsupervised dimensionality reduction of time series," in IEEE Conference on Pattern Recognition, Aug 2010, pp. 161-164.

[29] A. Gonzalez, M. Hayashibe, and P. Fraisse, "Estimation of the center of mass with kinect and wii balance board," in Conference on Intelligent Robots and Systems, 2012, pp. 1023-1028.

[30] J. Shotton, R. Girshick, A. Fitzgibbon, T. Sharp, M. Cook, M. Finocchio, R. Moore, P. Kohli, A. Criminisi, A. Kipman, and A. Blake, "Efficient human pose estimation from single depth images," IEEE Transactions on Pattern Analysis and Machine Intelligence, vol. 99, p. 1, 2012.

[31] C. Cortes and V. Vapnik, "Support-vector networks," Machine Learning, vol. 20, no. 3, pp. 273 - 297, September 1995.

[32] L. Breiman, "Random forests," Machine Learning, vol. 25, no. 1, pp. 5 $-32,2001$.

[33] G. W. Taylor, G. E. Hinton, and S. Roweis, "Modeling human motion using binary latent variables," in Advances in Neural Information Processing Systems, 2006, pp. 1345-1352.

[34] Y. Freund and R. E. Schapire, "A short introduction to boosting," Journal of Japanese Society for Artificial Intelligence, vol. 15, no. 4, pp. 771$780,1999$.

[35] H. T., R. Tibshirani, and J. Friedman, The Elements of Statistical Learning, second edition, Ed. New York: Spriner, 2008.

[36] S. C., T. Khoshgoftaar, J. Hulse, and A. Napolitano, "Rusboost: Improving clasification performance when training data is skewed," in IEEE Conference on Pattern Recognition, 2008, pp. 1-4.

[37] M. Warmuth, J. Liao, and G. Ratsch, "Totally corrective boosting algorithms that maximize the margin," in Proc. 23rd Int'l. Conf. on Machine Learning, 2006, pp. 1001-1008.

[38] L. Breiman, "Bagging predictors," Machine Learning, vol. 26, pp. 123140,1996
[39] A. Amini Maghsoud Bigy, K. Banitsas, A. Badii, and J. Cosmas, "Recognition of postures and freezing of gait in parkinson's disease patients using microsoft kinect sensor," in IEEE Conference on Neural Engineering, 2015, pp. 731-734.

[40] R. N. Baumgartner, K. M. Koehler, D. Gallagher, L. Romero, S. B. Heymsfield, R. R. Ross, P. J. Garry, and R. F. Lindeman, "Epidemiology of sarcopenia among the elderly in new mexico," American Journal of Epidemiology, vol. 147, no. 8, pp. 755-763, 1998.

[41] R. M. Dodds, H. E. Syddall, R. Cooper, M. Benzeval, I. J. Deary, E. M Dennison, G. Der, C. R. Gale, H. M. Inskip, C. Jagger, T. B. Kirkwood, D. A. Lawlor, S. M. Robinson, J. M. Starr, A. Steptoe, K. Tilling, D. Kuh, C. Cooper, and A. A. Sayer, "Grip strength across the life course: Normative data from twelve british studies," PLoS One, 2014.

[42] D. Leightley, B. Li, M. H. Yap, J. McPhee, and J. Darby, "Exemplarbased human action recognition with template matching from a stream of motion capture," in Image Analysis and Recognition. Springer, 2014, pp. 12-20.

[43] J. Guralnik, E. Simonsick, L. Ferrucci, R. Glynn, L. Berkman, D. Blazer, P. Scherr, and R. Wallace, "A short physical performance battery assessing lower extremity function: Association with self-reported disability and prediction of mortality and nursing home admission," Gerontology, vol. 49, no. 2, pp. 85 - 93, March 1994. 